\title{
PROLEGÓMENOS PARA UNA REGULACIÓN INTERPERSONAL DEL RIESGO
}

\author{
Prolegomena for an Interpersonal Risk \\ REgULATION
}

\section{AleXander Vargas Tinoco*}

\begin{abstract}
Resumen:
El presente trabajo explora algunos argumentos que permitirían una responsabilización interpersonal por el riesgo generado y, consecuentemente, una regulación interpersonal de este a través de la responsabilidad civil. En ese sentido, se aborda una interpretación de la probabilidad que así lo permitiría, algunas implicaciones conceptuales del riesgo y la cuestión de la estructura normativa de la responsabilidad civil. Finalmente, se deja planteada una justificación para que este ámbito del derecho se ocupe auxiliarmente de la regulación del riesgo.
\end{abstract}

Palabras clave: riesgo; peligro; responsabilidad; responsabilidad civil; probabilidad; regulación

\begin{abstract}
This paper explores some arguments allowing the interpersonal liability for the risk generated and, consequently, an interpersonal risk regulation through tort law. In this sense, it deals with an interpretation that would allow this, some implications of the concept of risk and the question of the regulation system of the law of torts. Finally, a justification is given for this area of the law to deal with risk regulation in an auxiliary manner.
\end{abstract}

Keywords: Risk; Danger; Responsibility; Tort Law; Probability; Regulation

* Universidad Externado de Colombia (alexander.vargas@uexternado.edu.co). Miembro del Proyecto de Análisis Filosófico del Derecho Privado de la Cátedra de Cultura Jurídica. Agradezco a Esteban Pereira Fredes, Alberto Pino Emhart, Sebastián Figueroa Rubio, Sebastián Agüero Sanjuán, Lucila Fernández Alle, Jouline Ropero y Joaquín Reyes por comentarios previos a una versión primigenia de este trabajo, que fue presentado en el Special Workshop 96 (Philosohy of Private Law) organizado en el marco del Congreso Mundial de Filosofía del Derecho (IVR) celebrado en julio de 2019 en Lucerna, Suiza. Artículo recibido el 9 de junio de 2020, y aceptado para su publicación el 1 de julio de 2020. 


\section{INTRODUCCIÓN}

La literatura en torno al riesgo, tanto en el ámbito jurídico como en el que no, es amplia y compleja de esquematizar respecto de los problemas que aborda. La dificultad aumenta cuando uno se percata de que las perspectivas de otras áreas (como la psicología, sociología, epidemiología, estadística, entre otras) pueden tener incidencia directa en muchas de nuestras apreciaciones jurídicas sobre el riesgo. Sorprende lo poco que los juristas creen poder hacer respecto del riesgo como tal, más allá de regularlo estatutariamente mediante el derecho público (como una cuestión de la que se deben ocupar otros expertos) o reparando el daño que se derivaría de él.

En el presente escrito pretendo introducir algunos argumentos que permitirían una tercera vía, cual es la de una regulación interpersonal del riesgo. En ese orden, me referiré a cuatro argumentos a partir de los cuales puede discutirse la posibilidad de responsabilizarnos interpersonalmente por el riesgo que imponemos en nuestras interacciones con otros. ${ }^{1}$ Lo anterior implica que alguien pueda llamar a cuentas a otro por la generación de ciertos riesgos y, en esa medida, pueda haber una regulación interpersonal de este que podría ser paralela a la regulación estatutaria. ${ }^{2}$ Cada uno de los argumentos también puede ser entendido como un escenario de debate teórico respecto de la posibilidad en comento, pues detrás de cada uno de ellos hay importantes divergencias cuyos términos no me es factible abordar a plenitud, y mucho menos resolver. Me limitaré a exponer cómo cada uno de ellos podría aportar, y en qué medida, a defender una posible regulación interpersonal del riesgo, de la cual supongo como condición la posibilidad de responsabilizar a alguien en particular.

El objetivo general no es otro que el de explorar los términos interdisciplinarios iniciales en los que puede darse esta discusión, y despertar un tanto más el interés sobre este asunto que no ha tenido un rol protagónico en el ámbito teórico-jurídico. Así, todos los argumentos serán presentados como "posibili-

1 Pueden admitirse dos sentidos en los que se puede entender algo como "interpersonal". Un sentido amplio refiere a aquello que involucra una relación entre dos o más personas, o entre una persona y todas las demás. Un sentido más estricto, al que me referiré principalmente, se puede entender como sinónimo de bilateralidad o bipolaridad, esto es, la vinculación de dos extremos entre sí que tienen posiciones normativas correlativas y especiales en esa relación. Al respecto, Darwall (2013a), pp. 12-15 y 20-39.

2 Sobre la idea de responsabilizar como "llamar a cuentas" se puede leer FIGUEROA (2019). Entiendo por "regulación interpersonal" aquella que no necesariamente es el resultado de prescripciones estatutarias o reglamentarias dictadas por los órganos administrativos o legislativos, sino la que resulta de regular una interacción entre dos extremos mediante normas genéricas de razonabilidad de la conducta. 
dades" para la defensa de la tesis y su presentación será como sigue. El primer argumento examina una interpretación de la probabilidad que permitiría la responsabilización interpersonal por la producción de riesgo. El segundo aborda cierta conexión conceptual que se ha sugerido entre el riesgo y la responsabilidad. El tercero se refiere a la posibilidad de que el derecho de la responsabilidad civil sea una institución adecuada para canalizar tal responsabilización y, finalmente, como cuarto argumento, expondré una posible justificación que haría plausible que se adopte la propuesta del tercer argumento.

\section{LA POSIBILIDAD INTERPRETATIVA}

Conectada a la idea de riesgo está la de posibilidad y, en ese orden, la de probabilidad, porque la determinación de cuán posible es un evento nos lleva a los terrenos de esta. Así, identificar una comprensión de la probabilidad que permita la responsabilidad interpersonal representa un primer reto. ${ }^{3}$ La cuestión interesa en la medida en que alguien pueda interpretar que la incertidumbre implícita en el concepto de riesgo es suficiente para rechazar que este pueda llegar a fundamentar un juicio moral razonable en contra de alguien. Podría considerarse que aquello que concebimos como riesgo no alude más que a meras estimaciones especulativas respecto eventos futuros (por definición no acontecidos) que, como tales, no alcanzarían para fundamentar un juicio de valor respecto de lo que alguien "ha hecho" o puede llegar a producir efectivamente en el mundo. ${ }^{4}$

En esta línea, no sería plausible responsabilizar por aquello que implica una reserva, duda o ignorancia respecto de lo que sucederá o ha sucedido. ${ }^{5}$ Este tipo de reparo supondría que (i) nuestros juicios morales deben fundarse en creencias justificadas en la realidad del mundo y que (ii) referirse al riesgo es una forma de expresar un desconocimiento sobre la producción de un resultado, en tanto implica una forma de incertidumbre. ${ }^{6}$ Así, la deficiencia epistémica del sujeto sobre la realidad, que parece constitutiva del concepto mismo, revelaría una forma de ignorancia con base en la cual nadie debería ser responsabilizado. Cuando se habla de resultados como el daño, en cambio, uno podría ser juzgado por nuestra incidencia en el mundo y la afectación que produjimos en él.

3 OberdieK (2017), p. 46.

4 Wright (2008), p. 1296, n.p. 6; RenN (1992).

5 Toulmin (1958) citado en Olivé (1981).

6 BAmmer et al. (2008), p. 298 y Riesch (2012). 
A lo anterior se suma que, desde ciertas perspectivas de la probabilidad, el riesgo dependería de la disposición del individuo para convencerse de algo, en este caso, de la ocurrencia futura de un resultado. ${ }^{7} \mathrm{Si}$ además se asume que los resultados esperables en el mundo son determinados por leyes causales y no por las convicciones o grados de convencimiento de las personas, el riesgo aparece como una base poco fiable para la responsabilidad. ${ }^{8}$ En efecto, para quien asume una postura determinista de los sucesos, todas las formas de incertidumbre, incluyendo la probabilidad, serán una expresión de las dificultades epistémicas del sujeto, no corresponderán necesariamente a la realidad de lo que sucede en el mundo $\mathrm{y}$, en ese orden, los juicios morales solo podrían dirigirse contra la creencia de la persona sobre sus actos y no contra su acción en sí misma considerada. ${ }^{9}$ Así, resultaría carente de sentido darle una relevancia moral al riesgo per se, dado que este se fundamenta en dificultades epistémicas y no en certezas que expliquen adecuadamente la causa de los eventos. ${ }^{10}$

Como puede notarse, buena parte de la dificultad anotada radica en la manera en que se interpreta la probabilidad que aparece implícita en el concepto y en el hecho mismo de que la probabilidad comporte alguna forma de ignorancia. Si la probabilidad es una cuestión subjetiva que solo da cuenta del estado del conocimiento de la persona (su "opinio"), al menos habría que justificar por qué se ha de responder por ella, a pesar de no ser necesariamente descriptiva del mundo. Respecto de esto, es bien sabido que históricamente ha habido una dualidad en la interpretación de la probabilidad, según la cual se le ha concebido, o bien como (i) una forma de expresar el grado de creencia en ciertas proposiciones desprovistas de antecedentes estadísticos, o bien como (ii) un concepto estadístico que se refiere a las leyes estocásticas sobre los procesos que involucran alguna forma de azar y cómo de ellos pueden pronosticarse ciertos resultados. ${ }^{11}$ Así, en contraposición a la concepcion subjetiva de la probabilidad, hay interpretaciones que pretenden un alcance descriptivo (y predictivo) fiable de lo que acontece o acontecerá, ya sea apelando a la frecuencia con que se producen ciertos resultados en una serie

7 Sobre la evolución del concepto riesgo, Wood (1964), p. 91.

8 Para una crítica sobre la probabilidad como grado de convencimiento, Ferrer (2007).

9 Para un examen sobre las teorías sobre la verdad, HAACK (1982), pp. 112-115. Según Hurd (1994), pp. 200-201 y (1996), pp. 262-263, esta característica hace que lo que sea incorrecto o injusto sean nuestras creencias respecto de la corrección o incorrección de nuestras acciones y, en ese sentido, nuestra culpabilidad, más no hace que las acciones en sí mismas sean incorrectas, porque en un mundo determinado causalmente la acción riesgosa no genera ninguna posibilidad de daño.

10 Así también Moore (2011), p. 240 y Perry (1995), pp. 323-324.

11 Sobre esta dualidad véase HACKING (1975), p. 86 y ss., también HaACK (2014), pp. 56 y ss. 
de eventos posibles, o apelando a la propensión con la que se tiende a producir ese resultado. ${ }^{12}$ Este tipo de caracterizaciones se basan en el funcionamiento de modelos teóricos racionalmente estructurados, que buscan representar aspectos de la realidad aunque sea con base en algunos datos que se tienen de la totalidad de variables en juego, y cuyo conocimiento pleno permitiría la predicción exacta de un resultado. Con base en estos modelos, aunque no conozcamos con exactitud qué resultado se obtendrá de arrojar un dado sobre una mesa en condiciones normales, porque para ello tendrían que conocerse todas las variables relevantes (fuerza del lanzamiento, altura, dirección, tamaño, peso, proporción, dureza del dado y de la mesa, resistencia de esta, del aire, entre otras), podemos tomar en cuenta algunos aspectos conocidos (como el hecho de que solo hay 6 resultados posibles, del 1 al 6) para determinar cuán posible es que se produzca cierto resultado. ${ }^{13}$ En este sentido, la probabilidad de un evento ya no variará según la actitud o disposición del sujeto, sino según el modelo teórico al que se haya acudido racionalmente para determinarla.

Así, si se entiende que el riesgo es un concepto que involucra la posibilidad de un resultado en el mundo, entonces en su determinación debería haber un desiderátum de objetividad y correspondencia con este, que haga que su medición solo pueda variar en función del conocimiento que progresivamente se adquiera de él. ${ }^{14} \mathrm{La}$ intersubjetividad que ofrezca el modelo, en tanto racionalmente construido, permitiría contrarrestar la arbitrariedad de las proposiciones que sobre la probabilidad de un evento se puedan efectuar, pues los individuos racionales se verán comprometidos a aceptar como verdaderas las conclusiones que se obtengan bajo el método válido que fundamente el modelo. De esta manera, la apelación al riesgo no será sinónimo de mera especulación o total ignorancia, sino de conocimiento sobre lo que podríamos esperar de la realidad a partir de un modelo de razonamiento basado en conocimientos parciales sobre un fenómeno. ${ }^{15}$ Este

12 Para diferentes interpretaciones de la probabilidad, HÁJEK (2019).

13 Olıvé (1981), p. 39.

14 Este tipo de respuesta no resultaría plenamente satisfactoria para quienes son escépticos de la posibilidad de escindir los elementos subjetivos de las cuantificaciones que pretenden alguna objetividad bajo modelos teóricos que se valen de mediciones numéricas. En este sentido, se ha dicho que puede haber valoraciones implícitas incluso en la misma determinación de riesgo. Al respecto, Hansson (2007). En esa línea, hay quienes mantienen que es un error incurrir en una "cosificación" del riesgo, esto es, abordarle como un concepto ajeno a factores subjetivos que tienen que ver con la psicología o la valoración personal de quien lo determina. Al respecto, TAYLOR (2012), p. 447.

15 En este sentido, el riesgo aportaría un conocimiento en forma de cuantificación. De hecho, hay quienes afirman que la cuantificación de la probabilidad es el carácter distintivo del riesgo respecto de la mera incertidumbre. Al respecto, KNIGHT (1921) o SunSTEIN (2005), p. 87. 
modelo, en tanto se fundamente en evidencias sobre el funcionamiento del mundo que permitan inducir ciertas conclusiones racionales sobre la posibilidad de los resultados, será accesible a quien responsabiliza y a quien es responsabilizado, incluso cuando el acceso a él pueda requerir algún esfuerzo de las partes de una interacción. ${ }^{16}$ Así, el riesgo con base en el cual vale la pena responsabilizar a otro es aquel que constituye un conocimiento aunque sea parcial del mundo con el que nos relacionamos y que puede servir de base para justificar la responsabilización de alguien por su generación.

\section{LA POSIBILIDAD CONCEPTUAL}

Adicionalmente, podría notarse que, no solamente la noción de probabilidad que se relaciona a la de riesgo puede tener una interpretación que permita la interpelación entre las personas y su eventual responsabilidad. El mismo concepto, al menos en un nivel muy general, parece implicar un carácter valorativo inescapable que permite vincularlo a la idea de responsabilidad.

Para evidenciar cómo, habría que notar primero que el riesgo no se trata de un concepto que flota en el aire, sino que implica la posibilidad de un resultado que afectaría algo o a alguien dentro de un ámbito temporal, locativo y/o material determinado o determinable. De no ser así, nuestra incertidumbre sería plena y toda acción para contrarrestar el posible resultado estaría injustificada. De hecho, si se observa con atención, la idea de una "clase de referencia" en las tesis frecuentistas sobre la probabilidad es posible gracias a que el ámbito del riesgo puede ser delimitado de alguna manera, por lo que la determinación del riesgo se identifica con la de las personas u objetos sometidos a él de cierta manera. ${ }^{17}$ Este ámbito de afectación no está desprovisto de una relación con un sujeto. Por el contrario, la referencia al riesgo conlleva que el resultado posible ha sido valorado por alguien como indeseable. Teniendo en cuenta esto, se podría sugerir una relación conceptual, aunque sea tenue, entre el riesgo y la responsabilidad. La relación no parece evidente, pero si se examina lo que implica que el riesgo comporte la indeseabilidad de un resultado que se estima negativo, entonces se aprecia un elemento valorativo dentro del concepto que apunta a la presencia de razones para que no se produzca ese resultado. ${ }^{18}$ Dicho de otro modo, la indeseabilidad de tal resultado implicaría la existencia de razones para la acción que

16 Al respecto, Kumar (2015), pp. 46-7, y OberdieK (2017), p. 56.

17 Un debate sobre lo que ello implica para el contractualismo se da en KUMAR (2015), p. 45 .

$18 \mathrm{Al}$ respecto, véase Hansson (2018). 
son discernibles por un sujeto que lo valora. Dichas razones pueden ser dirigidas por el sujeto valorador contra quien pueda discernirlas también, quien por su vinculación con el riesgo podrá actuar de cierta manera ante las razones que se le transmitan. Así, la probabilidad de un resultado indeseable supone la posibilidad de una dirección de razones respecto de ese resultado y, en esa medida, permite conceptualmente una responsabilización, aunque sea de uno mismo, para hacer algo ante el riesgo valorado. ${ }^{19}$

Lo anterior supondría que, según nuestros conocimientos, ese alguien a quien se dirigen las razones tiene alguna relación de control, aunque sea limitada, con la posible producción de un resultado indeseable. De no ser así, parecería infundada la responsabilización que supone el reclamo. Esas posibilidades de actuación del sujeto respecto del riesgo pueden tener una proyección retrospectiva, una vez producido el resultado, o también prospectiva, antes de que el resultado se produzca. ${ }^{20}$ En ambos sentidos en los que se proyecte la responsabilidad es posible la dirección de reclamos interpersonales que provean razones para adoptar ciertas acciones relacionadas con esa probabilidad que se genera para otros. Esta posibilidad de dirigir demandas que suponen una responsabilización permite ubicar el riesgo en el ámbito de una moralidad construida interpersonalmente, que se ocupa de todo aquello que provea para un reclamo válido hacia otro. Así, que alguien pueda tener un reclamo subyacente en contra de la imposición o la generación del riesgo muestra la relevancia que este puede tener en un contexto en el que lo moralmente obligatorio se compone de aquello que nos podemos exigir unos a otros respecto de nuestras conductas. ${ }^{21}$ Así, el riesgo parece ser un concepto híbrido que involucra un conocimiento (aunque incompleto) de ciertas relaciones causa-efecto, y un mensaje de índole prescriptiva para que "alguien" haga algo respecto de la producción de esos efectos que se consideran indeseables. ${ }^{22}$

Nótese que lo anterior no supone la superación de la crítica de la ignorancia de la causalidad que puede erigir el determinismo respecto de la idea de probabilidad (abordada en el acápite anterior), pero cuestiona la necesidad del conocimiento causal pleno como condición necesaria para la responsabilización de alguien. El desideratum del conocimiento causal pleno sería necesario solo si se concibe que nuestras prácticas morales versan exclusivamente sobre nuestros

19 VAn de Poen y Nihlén (2018), p. 885.

20 Van de Poen y Nihlén (2018), p. 886.

21 En general, Darwall (2006) y Wallace (2019), p. 62.

22 RenN (1992), p. 58. También Beck (1992), pp. 33-34, anota que el riesgo implica algo real e irreal al mismo tiempo, refiriéndose a estos aspectos descriptivos y a la amenaza que comportan. 
resultados en el mundo, sin que sean relevantes otras cuestiones como nuestras actitudes reactivas. Ciertamente, nuestras reacciones hacia otros juegan un rol importante que permite demandar algo de los demás, incluso en ausencia de resultados, por lo que no debería restársele toda importancia a ellas debido a la parcialidad de nuestro conocimiento causal.

Con todo, aunque responsabilizar a alguien en el sentido más básico requeriría solamente del reconocimiento del otro como alguien que puede ser destinatario de un reclamo implícito en una actitud reactiva, ${ }^{23}$ como seres racionales consideramos necesaria una justificación coherente que puede transmitir razones a otro. Por ello, aunque no sea necesario un conocimiento pleno de la causalidad para responsabilizar, la justificación para el reclamo por el riesgo puede venir dada por una estimación de las probabilidades que se haya dado de la manera más objetiva o intersubjetivamente controlable que estimemos, según se vio antes, mediante la adopción de un modelo teórico fundamentado en hechos o evidencias del mundo, y del que se pueden extraer conclusiones racionalmente válidas. ${ }^{24}$ Para tal efecto, es necesario que las personas cuenten con las capacidades inductivas necesarias para realizar tales estimaciones, aun cuando estas puedan ser complementadas o corregidas posteriormente con conocimientos más exactos que provengan de un modelo teórico más completo. ${ }^{25}$

\subsection{La distinción riesgo-peligro y la necesidad normativa}

Ahora, también desde la sociología se ha planteado una interesante conexión entre riesgo y la responsabilidad, no a partir del carácter indeseable del resultado, sino del acaecimiento del resultado y nuestra capacidad decisoria. En esa línea, en tanto se conciba la capacidad de decisión como uno de los presupuestos de la

23 Al respecto, Strawson (1995), pp. 41-53.

24 Las conclusiones acerca de la probabilidad así obtenidas son denominadas "probabilidad epistémica" por Perry (1995), p. 325.

25 Que exista un modelo para representarnos lo que puede suceder en el futuro no implica una descripción plena de la realidad ni un vínculo causa-efecto entre aquello que aumenta la posibilidad del resultado y el resultado mismo. Esto podría generar una objeción moral por ser responsabilizado por lo que no necesariamente pasó o pasará, pero incluso esta objeción no es suficiente para negar que la generación de riesgo pueda ser moralmente significativa en sí misma considerada como para justificar una reacción en contra. De hecho, si exigiéramos un conocimiento causal completo para nuestros juicios de responsabilidad civil por daños, difícilmente podríamos llevar a cabo dicha práctica jurídica. Los jueces y litigantes no siempre tienen acceso a la totalidad de datos relevantes sobre lo que causó un accidente, por lo que se centran en fundamentar probatoriamente cierta hipótesis que pretende explicar la causalidad de lo ocurrido. 
responsabilidad, esta podría predicarse de quienes deciden respecto de un posible resultado dañoso. Luhmann, por ejemplo, afirma que la diferencia entre el riesgo y el peligro se halla en que, en el primero, "los posibles daños futuros son atribuibles a la propia decisión", como cuando uno está en riesgo de estrellarse en un avión solamente si ha decidido previamente subirse a él. ${ }^{26}$ En cambio, en el caso de los peligros, "el daño tiene una causa externa. Así ocurre si, por seguir con el ejemplo dado, a uno le matan los restos de un avión que cae". ${ }^{27}$ En este sentido, Giddens afirma que "[1] que trae al juego la noción de responsabilidad es que alguien toma una decisión que tiene consecuencias discernibles". ${ }^{28}$

Esta distinción, no obstante, parece tener sus propias dificultades. Por un lado, habría que especificar cuáles son las condiciones bajo las cuales se entiende que una elección sobre el daño posible pertenece al ámbito de decisión del sujeto y no es externa a él. Por ejemplo, si se entendiese por decisión la mera elección entre dos alternativas, uno puede considerar que en los peligros también mediarían decisiones de alguien. Imaginemos que una persona conduce su coche y repentinamente una avalancha sepulta su vehículo: aunque se podría pensar que el daño provino de una fuente externa a la decisión del sujeto, no podría negarse que la elección del conductor de pasar por allí fue causalmente necesaria para ser sepultado. En un sentido no trivial todas nuestras elecciones sobre las alternativas disponibles se vinculan con el riesgo si se describen como condiciones necesarias para la producción del daño.

Por otra parte, parece que un observador de segundo grado tendría que determinar cuál es la decisión relevante en juego para identificar si se trata de un riesgo o de un peligro, pues puede haber decisiones externas a uno que sean internas a otros. ${ }^{29}$ Piénsese que el propietario del predio donde se produjo la avalancha decidió no advertir el "peligro" a los transeúntes de la zona. Habría una decisión en juego, pero no sería solamente la de la persona afectada por el resultado, de manera que habría dos riesgos para ambos, lo cual no dice nada sobre quién debe actuar para moderarlo o asumir el daño que eventualmente suceda. En otras palabras, los términos "externos" en que se puede describir el peligro también son reconducibles a términos internos en que se describe el riesgo.

26 Luhmann (1991), p. 81. Citado en Beck (1999), p. 132.

27 LuHMANn (1991), p. 81. Citado en BeCK (1999), p. 132. En sentido similar, LuHMANN (1993), p. 21. BeCK (1992), p. 121, secunda esta idea al afirmar que en la sociedad del riesgo, este no puede atribuirse a algo externo, sino que los riesgos dependen de las decisiones de las personas y en esa medida pueden ser objeto de reflexión.

28 GidDENs (1999), p. 8.

29 LuHMann (1993), p. 26. 
Además, vincular el concepto de riesgo a la decisión de uno parece esconder la carga de una responsabilidad moral por las decisiones propias. Si se extrajera un principio de esta conexión, se diría que el discernimiento de las consecuencias por parte de un agente decisor haría que la responsabilidad por la producción de los resultados probables recaiga sobre él, en tanto sea él quien controle sus decisiones y su conducta respecto de lo que puede producirse. ${ }^{30} \mathrm{In}$ cluso si se afirma que lo "discernible" -o previsible- debería ser aquello que con cierta probabilidad ocurrirá cuando se elige cierto curso de acción, no siempre es el caso que respondemos por lo que podemos discernir o hemos discernido. Nuestras prácticas jurídicas de adjudicación se nutren de un contexto normativo que permite que no siempre el discernimiento o la decisión propia sean suficientes para la responsabilidad. Así por ejemplo, no siempre el resultado más fácilmente discernible es el que determina la responsabilidad de alguien. Existen resultados posibles que aunque sean poco probables pueden ser adjudicados a alguien para que responda por ellos. Es decir, no siempre los resultados normativamente relevantes por los que alguien responde son los más fácilmente esperables o previsibles de una actividad. Una aerolínea podría ser considerada exclusivamente responsable de la muerte de un pasajero por un accidente aéreo, aun cuando la probabilidad de accidente sea menor a la de un viaje exitoso, y aun cuando aquella probabilidad haya sido concebida por el pasajero. O también, el discernimiento de las consecuencias o resultados nocivos posibles no implica que su producción conlleve la responsabilidad por igual de las personas que los prevén, por el solo hecho de concebirlos previamente. Si paso por cierta calle a ciertas horas de la noche, sabiendo que es muy probable que me atraquen, no por ello soy responsable del atraco.

El punto es que, si bien el concepto de riesgo permite la responsabilización porque implica la presencia de razones dirigibles, no es suficiente en sí mismo para determinar la responsabilidad de alguien en particular. Así, se revela la necesidad de un contexto normativo a partir del cual se puedan concretar los términos en que alguien puede llegar a responder por el posible resultado sobre el que decide. Dicho de otra manera, la determinación de la responsabilidad por un riesgo que parece asumirse o imponerse no puede apoyarse meramente en términos causales que estimen como equivalentes todos los aportes para la producción del resultado. Dicho sea de paso, ello eliminaría la distinción interior-exterior en la que se apoya la distinción sociológica entre riesgo y peligro. Para que haya

30 En esta medida, y por virtud de esa misma autonomía, las personas deberían contar con la información y las capacidades suficientes para tomar decisiones coherentes con sus deseos de primer y segundo orden. Así, podría afirmarse que una forma de incorrección primaria en el ámbito de los riesgos estaría en no suministrar la información suficiente sobre este, sobretodo cuando no es fácil para el sujeto decisor común discernir los posibles resultados. 
responsabilidad por el riesgo se requiere que haya un marco normativo de fondo que permita cualificar la conducta de alguien, no solo como una que podría dar lugar a un resultado dañoso en términos causales, sino como una conducta que normativamente es relevante respecto de las demás en juego. Esto explicaría que el riesgo sea usado como criterio de imputación en la práctica jurídica y no como forma de examen de la causalidad (aunque estén íntimamente relacionados). Sin un contexto valorativo parece imposible determinar cuál es la conducta que se considera riesgosa en una interacción, y sin normatividad alguna también lo es determinar si el riesgo es irracional, inaceptable o si cierta persona debe responder por su generación o por su eventual acaecimiento. ${ }^{31}$ La mera decisión no bastaría para adjudicar una responsabilidad jurídica, sino que se requiere de una normación sobre las decisiones que se considerarán relevantes en el ámbito de los riesgos para efectos de la adjudicación de responsabilidad. ${ }^{32}$

\section{LA POSIBILIDAD ESTRUCTURAL-NORMATIVA}

Visto lo anterior, surge la cuestión de si la estructura normativa que atienda un reclamo por la imposición del riesgo debe ser una interpersonal, a la manera como se implementa el derecho de la responsabilidad civil, o si por el contrario otros ámbitos del derecho son más aptos para ello.

El "asunto preventivo" es, tal vez, uno de los debates más interesantes que surgen en este ámbito del derecho. Mientras que algunas tesis afirman que no dañar de ciertas maneras puede interpretarse como prioritario $^{33}$ o como ideal

31 WolfF (2006), p. 410.

32 Véase Galindo (2015), p. 155. En este sentido, el mismo Luhmann afirma que la distinción riesgo-peligro es exitosa solo en tanto haya un observador de segundo grado que atribuya la responsabilidad a alguien por haber decidido sobre el posible daño futuro. Uno diría, sin el ánimo de pretender establecer un marco normativo exhaustivo para la responsabilización, que no solamente la posibilidad de que las partes disciernan las consecuencias posibles incide en la determinación de la responsabilidad, sino también otros factores. Sin ánimo de taxatividad, algunos de ellos serían (i) las condiciones de libertad y consciencia en que toman decisiones sobre los riesgos que crean o asumen, (ii) el valor de las actividades o los resultados finales respecto de las cuales el riesgo es un medio para conseguirlos, (iii) la distribución de la información al momento de las interacciones riesgosas, (iv) la capacidad de controlar la producción de los resultados posibles y (v) las cargas y beneficios que impone la mera permisión o prohibición de las conductas riesgosas que cada parte puede llevar a cabo.

33 Keating (2018). 
normativo en este ámbito, ${ }^{34}$ otras sostienen que el objeto primordial de esta práctica se limita a hacerse cargo de los daños causados, negando así que haya tal cosa como un deber de no imponer riesgo a otros. ${ }^{35}$

Solo la primera de estas perspectivas parecería ocuparse directamente del riesgo como tal, pero si se ahonda un poco más en su fundamentación se puede ver que se trataría de argumentos asociados a la proximidad temporal del daño. Ello no quiere decir que en principio el efecto práctico de la respuesta preventiva no sea idéntico al efecto que tendría una regulación interpersonal del riesgo, sino que los fundamentos para una y otra pueden diferenciarse. ${ }^{36}$ En el caso de la prevención del daño, lo que se estimaría incorrecto es que el daño acontezca, por lo que se debería evitar su producción cuando esté próxima a ocurrir. Se justificaría una respuesta preventiva solo cuando es probable la ocurrencia del daño en un período que apremie a tomar medidas tendientes a su evitación. En esa línea no sería contradictorio decir que, aunque la responsabilidad civil por lo general tiene un papel regulador del riesgo ex post al daño, excepcionalmente puede intervenir cuando resulta ser muy probable que este ocurra. ${ }^{37}$ Así, aun cuando se afirme que la responsabilidad civil está llamada a ocuparse de la indemnización de los daños, pueden evidenciarse razones para evitar su producción. Estas razones pueden ser de índole económica o apelar a cierto ideal de justicia o moralidad.

Dentro de los argumentos económicos estaría el mismo hecho de que el sistema de responsabilidad civil representa un incentivo para que los sujetos racionales se abstengan de asumir una externalidad negativa en la medida en que sea eficiente hacerlo. ${ }^{38}$ En esta línea, una determinación de las situaciones en las que el daño puede ser evitado de manera eficiente, debería conllevar a evitar la producción de la externalidad en primer lugar y así lograr mayores beneficios. ${ }^{39}$

También, con argumentos de otra naturaleza se podría sostener que la prevención es una forma en que el derecho de daños es coherente, si es que conside-

34 Interpretaciones de la responsabilidad civil con un sentido protector ya han sido ofrecidas por SHeINMAN (2003) y PAPAYANnis (2014), p. 130.

35 Nolan (2013). Para perspectivas remediales del derecho de daños véase, entre otros, Coleman (1992), Koziol (2017) y Priel (2018).

36 Al respecto, Priel (2018), § 14 y $§ 18$, anota que la cuestión sobre los riesgos admisibles difiere de la cuestión sobre quién carga con las pérdidas que se producen.

37 Papayannis (2016), pp. 85-86.

38 ACCIARRI (2013), p. 430; TICHÝ (2013), p. 22.

39 Una exposición sobre la lógica económica de las prevenciones y la negligencia puede verse en POSNer (1972), pp. 31-33. 
ramos que esta rama del derecho se ocupa de distribuir derechos y deberes de no dañar (de ciertas maneras) entre las personas. En ese orden, si el ideal normativo de la responsabilidad es no dañar en ciertas maneras, la manera más coherente de alcanzarlo es evitando que el daño ocurra, no esperando a que acontezca para indemnizarlo. Así, detrás de cada derecho a no ser dañado, culposamente o por una acción riesgosa, habría razones para evitar el daño, porque cuando se previene se refuerza un derecho, no contra el riesgo, sino contra el daño. Como bien se sabe, parte de lo que comprende tener un derecho es la posibilidad de que este sea reforzado institucionalmente, de manera que se evite su infracción o se provea una reparación o una sanción en caso de ser infringido. ${ }^{40}$ De fondo, uno podría apreciar un argumento moral aquí -también reconstruible en términos conceptuales- según el cual, si es cierto que el sujeto tiene este tipo de derechos de indemnidad, entonces tiene una forma de autoridad individual ante los demás con base en la cual puede exigir respeto. ${ }^{41}$ Con base en dicha autoridad, al individuo se le debe una abstención de la infracción de sus derechos primarios, dado que el valor de su autoridad no puede ser reducido al del dinero que le pueda proveer con una indemnización por el daño sufrido injustamente. Esta apreciación consideraría que parte de la autonomía es poder pretender autoritativamente algo de los demás, lo cual implicaría una forma de inconmensurabilidad de aquella que no puede ser restituida con los remedios monetarios. Dicho de otro modo, tal como inteligimos el respeto, este se trata de un concepto que requiere que el cumplimiento del deber hacia el objeto de respeto se produzca en primer lugar y no posteriormente, porque posteriormente ya se habrá perdido normativamente algo que hace que el respeto deba guardarse ab initio. Así, el respeto busca conservar el valor original de aquello que es el objeto del deber, en este caso, la indemnidad de la persona. ${ }^{42}$

Pero si se defiende que la regulación del riesgo es importante también para la responsabilidad civil o que lo significativo para este ámbito del derecho no solamente es el resultado daño, entonces los argumentos ya no son necesariamente los mismos. Existen riesgos que no nos interesa disminuir aunque nos puedan acarrear un daño indemnizable, y en esa medida las razones para una y otra acción serían diferentes. Además, cuando se dice que el riesgo es meritorio de una regulación per se, parece aludirse solo a una conducta que es normativamente relevante en tanto alejada de cierto estándar, incluso cuando no pueda afirmarse rotundamente que el daño está próximo a suceder. Es decir, que en la regulación del riesgo por el hecho de su imposición no necesariamente la falta

40 RAZ (1996), p. 256.

41 Darwall (2013b), p. 13.

42 Un argumento similar en VARgas-Tinoco (2018). 
de proximidad del daño restringe la provisión de una respuesta, pues también la magnitud del daño, aunque no sea uno inmediato, podría jugar a favor de una regulación. ${ }^{43}$

A diferencia de la generación del daño, donde se evidencia un resultado determinado del que uno debería hacerse cargo por haber afectado al mundo de esa manera, o por estar próximo a causarlo, en el caso del (mero) riesgo como fundamento de la reacción jurídica, lo relevante parecería ser la conducta misma, independiente de la producción fáctica de las consecuencias o su inmediatez temporal. Bajo este argumento, en ausencia de un resultado que perjudique a alguien en particular, alguien diría que no es posible ofrecer respuestas jurídicas interpersonales contra tipos de conductas en las que alguien puede incurrir y cualquiera podría ser "víctima" sin tener una conexión en particular. ${ }^{44}$ Así, ante la falta de un resultado no habría tampoco lugar a hablar de causalidad, por lo que no se presentarían los elementos básicos de la responsabilidad civil. ${ }^{45}$

No obstante, si la objeción de la ausencia de un resultado es básica para alegar una desconexión entre las partes de una interacción riesgosa, sus detractores también tendrían que lidiar con varios argumentos que intentan mostrar precisamente que alguien en particular puede resultar afectado negativamente cuando ha sido pasible del riesgo. ${ }^{46}$ Una forma de llegar a tal conclusión es argumentando a favor de un carácter dañoso de la imposición de riesgo, esto es, que en ciertas condiciones, ser pasible de un riesgo puede generar un daño que permite la vinculación entre las partes. Las vías para llegar a defender tal tesis, no obstante, han diferido.

Una primera perspectiva apela al hecho de que cierta probabilidad de daños graves puede generar resultados adversos en la salud mental de las personas, tales como niveles elevados de estrés, ansiedad o fobia por el posible advenimiento del daño, lo que a su turno se considera dañoso. En tales casos habría una vinculación entre las partes por el hecho del daño. ${ }^{47}$

43 Es bien sabido que en la cuantificación del riesgo tiene pertinencia no solo la probabilidad del daño, sino también la magnitud de este. Así por ejemplo el numeral 3 del artículo 5:101 de los Principios Europeos del Derecho de Daños señala que "[e]l riesgo de daño puede ser significativo en atención a la gravedad o a la probabilidad del mismo".

44 Weinrib (2017), p. 181-182; MoOre (2011), p. 78.

45 Véase Nolan (2013), Turton (2015).

46 Esta objeción se puede tomar como similar a la objeción acerca de la posibilidad misma de que la probabilidad pueda tener alguna significación moral, a la que antes me referí.

47 En ese sentido, véase Goldberg y Zipursky (2002), p. 1634. Sobre daños ocasionados por la imposición de riesgo suele discutirse el caso Ayers v. Jackson Township, donde la 
Otra aproximación pone el ojo sobre la representación mental del resultado en el agente que pone en riesgo a otro al realizar su conducta. Así, hay quienes afirman que cuando el riesgo es intencional y grave, se produce un daño en los intereses en la dignidad de la persona que fue objeto de tal intención dañosa. Esto parte de la premisa de que la persona no debe ser el objetivo de conductas con las que se busca causar daño. ${ }^{48}$ Esta perspectiva, no obstante, se limitaría a aquellos casos en los que hay un animus nocendi, con lo que se dejarían por fuera casos en los que, no habiendo tal intención, parece haber una afectación seria a la persona por el riesgo. Así, también se ha dicho que dejar librado al azar el resultado mismo, incluso aunque no haya intención, permitiría evidenciar un injusto. ${ }^{49}$

Por otra parte, otros enfoques se desligan un tanto más de las actitudes del agente que genera el riesgo y se centran en la significación que tiene este para quien es pasible de él. Por ejemplo, se ha apelado a las preferencias de las personas para indicar que cuando alguien pone en riesgo a otro, lo que hace es obrar en contra de sus intereses, entendidos como preferencias respecto de la situación en la que a uno le gustaría estar. Así, si las personas prefierieran estar en las clases de referencia o grupos que tienen menos probabilidad de ser dañados, resultarían dañados cuando se contrarían tales intereses al ser sometidos a riesgo. ${ }^{50}$

Pero también ha habido argumentos que apuntan a que la imposición del riesgo tiene la capacidad de dañar en un sentido inmaterial que tiene que ver con las opciones que son valiosas para el sujeto. Con base en la idea de la autonomía expuesta por Raz, ${ }^{51}$ se ha mantenido que el riesgo hace que las opciones que podrían hacer parte del ramillete de alternativas que un sujeto autónomo tendría ya no hagan parte de ese menú de opciones, porque normativamente ellas resultarán disvaliosas por el daño que pueden acarrear. En ese sentido, el riesgo conllevaría un daño inmaterial consistente en la eliminación de opciones valiosas para la persona. ${ }^{52}$

Corte Suprema de New Jersey concedió como remedio a favor de los demandantes los gastos de monitoreo médico para vigilar que los accionantes no desarrollaran cáncer, después de haber sido expuestos a desechos tóxicos por el demandado, quien hizo un manejo negligente de los residuos a su cargo.

48 Placani (2016).

49 Schroeder (1990), pp. 152 y ss.

50 Finkelstein (2003), pp. 973 y ss.

51 Raz (1986), pp. 370-372. Según Raz parte de lo que se comprende por ser autónomo es contar con opciones valiosas y diferentes respecto de las cuales una persona puede decidir.

52 OBERDIEK (2017), p. 88. 
Cada una de estas tesis puede tener sus propias dificultades explicativas, y de hecho se ha presentado un interesante debate entre algunos de sus defensores, no obstante, parece haber un punto de coincidencia en el hecho de que el riesgo también puede generar un retroceso en los intereses de alguien, sea en forma de opciones o de preferencias. De hecho, quienes niegan que el riesgo pueda ser un daño, no niegan que este retroceso se produzca, sino el carácter primario de los intereses que retroceden. Se ha dicho, por ejemplo, que el riesgo solo hace retroceder intereses secundarios de alguien, es decir, esos intereses que se explican derivativamente a partir de los intereses primarios, pero no se niega que haya una afectación de intereses que pueda generar un reclamo en particular hacia alguien, o que pueda tenerse un derecho contra otro por el retroceso de tales intereses. ${ }^{53}$ Ahora, que se califique de "secundario" resulta bastante discutible en la sociedad contemporánea en la que hay altos niveles de exposición al riesgo, y graves consecuencias asociadas a él. Es evidente que tenemos un interés en los riesgos a los que somos sometidos y más cuando no tenemos el control de ellos, y esto es así incluso aunque no nos percatemos de la imposición del riesgo o seamos afectados por la experiencia negativa de la imposición. ${ }^{54} \mathrm{La}$ afectación a tal interés puede considerarse lo suficientemente relevante como para reputarse injusta y generar un reclamo por imponer una carga que rompe el equilibrio normativo que haya entre las partes.

No es el propósito aquí sentar cuál es la significación que tiene el riesgo sobre alguien como para afirmar que su imposición puede generar un daño. Sin embargo, no debería desconocerse que el riesgo conlleva una significación tal en ciertas condiciones, que asimilamos su generación a la de un daño, o al menos a un resultado injusto respecto del cual podemos dirigir un reclamo a otro. Esta significación suele relacionarse con el razonamiento práctico de las personas y su sentido mismo de libertad. Si bien el riesgo no parece eliminar o inutilizar de facto los medios con los que cuentan las personas para ejercer su autonomía -y en esa medida podría diferenciarse del daño-, ello no implica que no generen una interferencia o interrupción normativa en la autonomía de ellas. La interrupción que produce es diferente, pues parece consistir en direccionar la conducta hacia ciertas opciones seguras que un hombre razonable debería adoptar si pretende conservar los medios con los que cuenta para manifestarse con libertad en el mundo. Como lo señala Raz, "la autonomía requiere una elección de bienes. Una elección entre un bien y un mal no es suficiente... la autonomía no puede ser alcanzada por una persona que cada acción y pensamiento tiene que ser desviado a la tarea de sobrevivir...". ${ }^{55}$ En esta medida, es evidente que tener que adoptar decisiones en aras a la protección restringe el ámbito de libertad de las personas y, en esa línea, su autonomía. ${ }^{56}$

53 Perry (2003), p. 1.307.

54 Al respecto, JAMES (2017), p. 6.

55 RAz (1986), p. 379.

56 Ahora, algunas dudas podría generar el hecho de que no todas las actitudes ante el riesgo sean iguales. Así como hay personas que son aversas al riesgo y que buscan evadirlo, 
Así las cosas, aún en el caso en que el efecto disvalioso que genere el riesgo no se identifique con un daño, ello no quiere decir que no se presente un injusto interpersonal, correlativo a las partes de la interacción. Dicho de otro modo, bien podría haber un resultado injusto aunque no haya daño, porque no solamente la protección contra el daño es el objeto exclusivo de los derechos y deberes correlativos que podemos tener. Como bien se ha señalado, no es la producción de una pérdida lo que activa la justicia entre las partes (aludiendo a la justicia correctiva), es un injusto normativo lo que resulta relevante. Solo de esta manera es comprensible que haya pérdidas que no sean injustas, e injusticias que no generen pérdidas (e.g. trespass, ${ }^{57}$ nuisance, ${ }^{58}$ assault $^{59} \mathrm{y}$ algunos casos que dan lugar a injunctions $\left.{ }^{60}\right)$, siendo estas úl-

también hay otras que son propensas a él o neutrales, que por lo mismo no verán alteradas sus decisiones ni la valoración de sus opciones por el hecho del riesgo. En esa medida, la significación del riesgo no puede ser igual para todos. Dicha observación, no obstante, no es exclusiva del riesgo. La percepción de que algo sea dañoso tampoco es compartida por todos, pero eso no evita que veamos su evitación como algo deseable o que, incluso, lleguemos a exigirnos esa evitación. Solo así se explican nuestros estándares de diligencia, pues la propia percepción del daño como algo deseable no puede suponerse compartida por quien será víctima del daño. Tenemos que apelar a una valoración intersubjetiva de lo que es correcto hacer respecto del daño para impedir que el criterio personal se imponga a otros. Por lo general, esa valoración es negativa respecto del daño. En esta línea, quien es propenso al riesgo puede confiar en que este no sucederá o terminará por afectarle, pero no parece razonable exigirle a otros que tengan esa misma confianza respecto de los riesgos que él genera.

57 El Restatement (Second) of Torts $§ 158$ afirma la producción de un ilícito civil por la invasión de la propiedad ajena, independientemente de si dicha acción causa un daño o no. Este ilícito daría ocasión al pago de una indemnización siquiera simbólica por parte del infractor a favor del propietario demandante.

58 Puede cometerse un ilícito civil (tort) objeto de responsabilidad civil por "molestias privadas" que se originan a pesar de que la conducta del demandado no se materialice en un daño. En Birmingham Development Company Ltd v. Tyler, el demandante argumentó que el muro del demandado ponía en riesgo de daño físico a los trabajadores que realizaban obras de construcción en su predio, de modo que esas obras no podían llevarse a cabo de manera segura. En los países del civil law, una acción que pretenda llevar a cabo tal prevención del riesgo se encuentra en la llamada "acción de daño contingente", dispuesta en varios códigos civiles de América Latina (artículos 2359 C.C. colombiano, 2333 C.C. chileno, 2236 CC. ecuatoriano y 2084 C.C. salvadoreño). Una interesante variación más genérica se encuentra en el C.C. argentino, que dispone una acción preventiva en su art. 1711. Por virtud de este tipo de acciones se pretende que quienes están amenazados por un riesgo puedan hacer cesar este mediante una acción civil. Al respecto véase DIEZ-SCHWERTER (2016).

59 Jurídicamente se afirma que si la conducta de alguien hace que otro tema violencia inminente en su contra, con la intención de provocar ese temor en él, se comete el ilícito civil de asalto. Estrictamente, no se require un riesgo real para cometer el asalto, pero en todo caso es cierto que el derecho de daños en el common law estima que la imposición intencional del riesgo que se transmite como violencia de daño inminente se encuentra proscrita y se estima como un ilícito del que se puede ocupar esta área del derecho (véase Mbasogo v. Logo Ltd).

60 Según la doctrina, una injunction u orden judicial puede decretarse preventivamente aunque ningún ilícito haya sucedido aún. Al respecto, BurRows (2004), p. 543. Jurispru- 
timas las que son relevantes según el trasfondo normativo que guía la relación. ${ }^{61} \mathrm{Si}$ esto es así, no habría lugar a afirmar que hay imposibilidad conceptual alguna para que una forma de justicia que supone una responsabilización interpersonal se ocupe también de las imposiciones de riesgo. ${ }^{62}$ En este sentido, bien se puede señalar que la correlatividad de un injusto no depende de la producción de una pérdida fáctica o daño, sino de que se haya producido una infracción normativa entre los dos extremos. Adicionalmente se tiene el argumento conceptual de que el riesgo no cae en el vacío sino que podemos inferir quiénes pueden llegar a ser los afectados y, a partir de tales conocimientos, vincular al extremo generador del riesgo con quienes sean pasibles de él, quienes podrían ver vulnerados eventualmente sus derechos contra el riesgo. En esta medida, la generación de cierto tipo de riesgos puede tener la estructura dikaiológica o bilateral necesaria en virtud de la cual A comete un injusto contra B al hacer $\mathrm{C}$ y no una forma impersonal de incorrección que consista en que A comete un injusto al hacer C. ${ }^{63}$ Así, quien es pasible del riesgo puede tener lo que Wallace llama una "posición privilegiada" para el reclamo en contra del generador de este, ${ }^{64}$ o una posición normativa individual que le permite exigir una obligación bipolar, dicho en otros términos. En virtud de esta posición normativa, quien es afectado por el riesgo tiene ciertas facultades ante quien le ha agraviado, de manera que puede ejercer discrecionalmente las acciones judiciales que tenga para hacer valer sus derechos interpersonalmente contra otro. ${ }^{65}$

Dicho esto, la afirmación de que el riesgo genera razones para que cualquiera haga un reclamo parece poco ajustada a los efectos normativos que el riesgo genera para quien estima valiosa su auto-conservación. ${ }^{66}$ Aunque en las

dencialmente, la afirmaciópn de esta regla puede encontrarse en Redland Bricks v. Morris (1970), Lloyd v. Symonds \& Ors Respondent (1998) y London Borough of Islington v. Margaret Elliott, Peter Morris (2012).

61 WeInRIB (2017), p. 150.

62 Curiosamente, WeINRIB (2017), pp. 181-182, se opone a afirmar que un derecho a la seguridad esté de acuerdo con una estructura de derechos kantiana como la que él utiliza. No obstante, también afirma que es el riesgo no permitido lo que marca la incorrección del daño que sucede (2016, pp. 150-152) y que puede haber injustos sin daño, por lo que su negación de los derechos a la seguridad parece requerir fundamentos adicionales.

63 Darwall (2013a), p. 31.

64 Wallace (2007), p. 29.

65 Darwall (2013a), p. 36.

66 Podría objetarse que, al ser normativas, este tipo de afectación solo se produce en quienes son adversos al riesgo, por lo que la extensión de un derecho contra el riesgo para todos en general implica alguna forma de paternalismo. Sin embargo, detrás de la acusación de paternalismo hay dos suposiciones que no parecen acertadas. La primera es que las personas que son pasibles de un riesgo tienen una gran variedad de opciones como para no optar por 
hipótesis de riesgo se pueda prescindir de la producción fáctica de un daño, el hecho de que el riesgo esté conceptualmente ligado a un resultado indeseado permite apelar a nuestros conocimentos causales sobre ese resultado posible para identificar quiénes pueden ser afectados por él, de manera que puede adoptarse un modelo racional que provea premisas válidas sobre la predictibilidad de los resultados de la conducta riesgosa. ${ }^{67}$ Así, aunque las consideraciones causales no sean suficientes para establecer la responsabilidad de alguien en particular por el riesgo, pueden usarse como base para determinar el ámbito de afectación normativo con el que se carga a otros por su imposición.

\section{UNA POSIBLE JUSTIFICACIÓN}

Ahora, si bien las cargas que una parte impone a otra con el riesgo son relevantes como para pensar en una conexión entre ellas que pueda ser considerada jurídicamente relevante, existen otras consideraciones que pueden moderar las pretensiones interpersonales que podamos tener. Por ejemplo, si bien es cierto que el alcance del riesgo puede ser determinable, la cantidad de personas sometidas a él puede ser tal que resulta económicamente menos gravoso que un representante de la sociedad (por ejemplo un organismo de carácter público) o

estar sujetas al riesgo. Esto evidentemente niega contextos en los que las opciones de seguridad y los costes de tomar opciones más seguras no están al alcance de la persona promedio (un argumento similar en Dorfman, 2014). Segundo, parecen suponer que la significación del daño, a diferencia del riesgo, no dependería de la aversión a este, lo cual es infundado. También quienes sufren daño pueden optar por renunciar a efectuar cualquier reclamo por este o incluso pueden llegar a considerarlo placentero y no por ello entenderíamos que el daño deja de ser significativo (al respecto, SHIFFrin 2012).

67 Evidentemente pueden identificarse dos escenarios al momento de hablar de causalidad y riesgo. El primero, ex ante al daño, supone la caracterización de una conducta como riesgosa en tanto apta para causar cierto resultado, conforme a nuestros conocimientos actuales (aunque sean parciales). El segundo, ex post al daño, puede suponer, o bien que el daño ha sido causado efectivamente por una conducta que se consideraba riesgosa porque el resultado causado era probable, o que existen dudas sobre el hecho de que la actividad hubiese sido la causa efectiva del resultado, pese a que se trata de uno previsible para la actividad. En los casos en que el nexo causal está en duda no puede afirmarse que el riesgo sea la causa necesaria del resultado. En efecto, si la causalidad es explicada en términos de necesidad de una condición que se considera causa, no sería cierto que $\mathrm{A}$, al aumentar el riesgo de que $\mathrm{B}$ sufra $\mathrm{C}$ por hacer D, A vaya a causar o haya causado $\mathrm{C}$ al hacer D. Ahora, debe tenerse en cuenta que no por el hecho de que D no sea causa de C, A no sea responsable ante B por hacer D y la afectación a los intereses que hacer $\mathrm{D}$ supone. Ha habido casos en los que se interpreta con mayor laxitud la exigencia de causalidad y se otorga peso a la incorrección de la conducta del demandado al aumentar el riesgo (e.g. Fairchild v. Glenhaven Funeral Services Ltd., o también McGhee v. National Coal Board). 
de los afectados (como por ejemplo una liga de consumidores) tengan la facultad de accionar judicialmente en contra del generador de riesgo.

No obstante, es posible diferenciar entre no darle una vía judicial directa a alguien para que pueda ejercer su derecho a cierto nivel de seguridad en razón a los costes de implementación, y negar directamente el derecho a cierta seguridad en razón a los costes de prevención. Solo esta segunda situación implica una rotunda negación de un derecho interpersonal contra el riesgo. Pero también podría haber argumentos concernientes a la propia moralidad que impidiesen un derecho particular contra el riesgo. Las tesis que apelan al contractualismo y centran su atención en las cargas intrapersonales que se derivan de la permisión o prohibición del riesgo también podrían concluir que en ciertos casos resulta excesiva una prohibición o moderación de este. Con todo, las tesis de esta índole favorecerían más la idea de un derecho correlativo contra ciertos riesgos, pues no necesariamente las cargas se analizan en términos de costes que se contrarrestan con beneficios, agregativamente. Puede ser el caso que una sola persona tenga fuertes razones para objetar la permisión de cierto riesgo y oponerse en términos suficientes que conlleven a su prohibición o moderación.

Por cierto, la perspectiva contractualista podría arrojar una respuesta a la crítica usual de que una prohibición del riesgo implica la prohibición de todo tipo de acciones o hace imposible la acción moral del ser humano. ${ }^{68}$ En primer lugar, un derecho contra el riesgo no es un derecho contra todo riesgo, sino contra aquellos que se desvían del estándar aceptable respecto de uno en sus propias circunstancias particulares. Segundo, la eventual prohibición de una conducta se predicaría de otras solo en la medida en que se trate de conductas que se derivan del mismo tipo de actividad, porque dentro de la reflexión contractualista también hay que considerar el valor y las ventajas que las actividades tienen en general para el ejercicio de nuestra libertad. En este orden, no es que deba prohibirse toda acción que suponga la posibilidad de muerte, por ejemplo, sino solo aquellas conductas que supongan esta posibilidad y que además hagan parte de una actividad que podría arrojar la misma clase de beneficios, distribuidos más o menos equitativamente, a través de un curso de acción menos peligroso. ${ }^{69}$

Sin embargo, el debate respecto de la legitimidad con la que los jueces obrarían si fueran ellos los que establecieran principios en torno a la permisión del riesgo es inevitable. Se diría, en ese sentido, que el legislador es el llamado

68 WeinRIB (2017), p. 181.

69 En particular KeAting (2003) explora algunos estándares de seguridad para el riesgo. Otros principios similares de diligencia pueden entontrarse en JAMES (2017), pp. 6-7. 
a confeccionar tales normas porque ha sido instituido para ello y porque podría estar mejor informado sobre los términos técnicos relevantes a la hora de regular el riesgo. Empero, ninguno de los dos argumentos es suficiente para desvirtuar la posibilidad judicial de distribución de un derecho (y de regulación del riesgo, en consecuencia).

Por una parte, es sabido que un sistema normativo que se reputa completo debería contar con un juez que tenga la autoridad suficiente como para crear una norma o interpretar las existentes de manera que pueda emitir una respuesta jurídica ante una situación no regulada expresamente. ${ }^{70}$ De esa manera, su facultad creadora de derecho no es exótica sino ajustada a la idea misma de un ordenamiento pleno. Así, podría decirse que la interpretación de una norma que proteja la integridad o la autonomía de las personas contra los actos o conductas de los demás podría ser fundamento para la distribución de un derecho tal.

Por otro lado, los procesos judiciales no son ajenos a la información técnica con base en la cual se llevaría a cabo una regulación del riesgo en un ente legislativo. Existen suficientes mecanismos de introducción de información especializada dentro de los procesos judiciales como para considerar que el juez podría contar con herramientas epistémicas suficientes para decidir sobre los términos en los que se está sometiendo a un riesgo a una persona. ${ }^{71} \mathrm{El} \mathrm{juez,} \mathrm{en} \mathrm{todo} \mathrm{caso,} \mathrm{no}$ tiene vedadas las razones a las que el legislador puede acceder. También él es un sujeto racional que puede informarse. Por supuesto, no pretendo aquí homologar la labor del legislador a la del juez, sino resaltar el papel distributivo que ya se ha afirmado que tiene respecto de las reglas de responsabilidad civil.

Claro, parece que en principio la confección de reglas de conducta en una sociedad se discutiría mejor entre mayorías o sus representantes, a fin de que todas las posibles objeciones sean puestas sobre la mesa. En líneas generales, esto apoyaría que, siendo tantos los factores en juego con la regulación del riesgo, el juez se apegue a lo que se haya normado previamente sobre el riesgo, dejando sus facultades creadoras solo para los casos de verdaderos vacíos normativos. Sin embargo, existen dos argumentos que reforzarían la idea de un control judicial sobre los riesgos que se dé con ocasión a las interacciones interpersonales. Se trata de argumentos que ya han sido expuestos desde la sociología y que pueden ser una base justificatoria para la idea de un derecho en contra de ciertas imposiciones de riesgo.

70 PaPAYANnis (2016), p. 221.

71 Al respecto, VÁzQUEZ (2015). 
El primero de ellos obedece a las circunstancias propias de la realidad contemporánea. Como bien se ha señalado desde la sociología, nuestra modernidad se caracteriza por estar bajo riesgos que son el producto de los procesos industriales -muchas veces desmedidos- que vivimos en una primera modernidad. ${ }^{72}$ La idea de estar viviendo bajo condiciones de riesgo constantes o de posibles daños severos o irreversibles determina un contexto en el que se justificaría no añadirnos mayores cargas a las que ya nos imponemos. Adicionalmente, ya no parece que seamos ignorantes sobre la manera en que podríamos lograr ciertos fines u obtener ciertos beneficios mediante el uso de técnicas o medios alternativos menos riesgosos, sino que nuestros avances en la ciencia también han permitido pensar en otras opciones menos riesgosas que debilitan la justificación de las más riesgosas.

En segundo lugar, en la distribución de derechos a nivel legislativo puede presentarse una grave desconexión entre los intereses de las personas sometidas al riesgo y el papel regulador que en principio tendrían los órganos representativos en una sociedad. La existencia de lobbies de grandes sectores económicos que ejercen presión a los legisladores y a los mecanismos de control, termina desenfocando el papel democrático que cumplen los representantes de las personas en las instituciones del poder público. Esto deslegitima su papel y justifica una mayor participación directa de los interesados en que se regule aquello que los afecta. Lamentablemente, no es difícil encontrar ejemplos de pactos entre el sector público y las empresas privadas para efectuar una "autorregulación" ineficaz del riesgo ${ }^{73}$ o también la omisión legislativa de regular un riesgo conocidamente devastador para quienes se expongan a él. ${ }^{74}$ Estas circunstancias

72 BECK (1992), pp. 10-15.

73 Ejemplo de un pacto privado de autorregulación se encuentra en el caso de los productores de bebidas azucaradas en Colombia, según el cual se comprometieron a adoptar prácticas de prevención para cierto tipo de consumidores [sobre esto: "Marcas de gaseosas y té restringen venta a menores" (mayo 19 de 2016). Portafolio, (recuperado de www.portafolio. co/negocios/empresas/empresas-firman-pacto-vender-gaseosas-escuelas-primarias-496073)]. Posteriormente, se ha venido denunciando el incumplimiento de dicho pacto [véase: "Empresas de bebidas azucaradas incumplen acuerdos de venta en colegios" (mayo 23 de 2019). Liga contra el silencio, recuperado de https://ligacontraelsilencio.com/2019/05/23/empresas-de-bebidas-azucaradas-incumplen-acuerdos-de-venta-en-colegios/]. Otras organizaciones no gubernamentales también han hecho similares denuncias y han solicitado la regulación del riesgo generado por los productores de estas bebidas [al respecto: "Pedimos implantar el etiquetado frontal de advertencias en alimentos y bebidas de Latinoamérica" ( 9 de agosto de 2019), De justicia, recuperado de https://www.dejusticia.org/pedimos-implantar-el-etiquetado-frontal-de-advertencias-en-alimentos-y-bebidas-de-latinoamerica/ ].

74 Otro ejemplo para el caso colombiano está en la prohibición del asbesto. A pesar de que desde 1978 se conocen los efectos cancerígenos de esta sustancia, y de que en otros 
manifiestan una forma de fracaso de la representatividad democrática de ciertos entes legisladores, que puede evidenciar una forma de abuso por parte del Estado al momento de regular suficientemente la seguridad que los ciudadanos le han confiado. En este orden, la responsabilidad civil parecería ser una herramienta mediante la cual se pueden realizar ajustes distributivos respecto de la regulación del riesgo, cuando el juez dirima los reclamos que se le presenten respecto de una interacción riesgosa injusta entre particulares. La distribución judicial de los derechos contribuiría a la regulación estatutaria del riesgo y permitiría una constante mejora de la razonabilidad de las normas por las cuales nos comportamos ante otros. ${ }^{75}$ Claro, si el juez tampoco es una institución fiable que puede ser un contrapeso para las otras ramas del poder, ningún ámbito del derecho podría suplir tan graves falencias democráticas.

países fue prohibido su uso desde finales del siglo pasado (Europa lo prohibió desde 1999 mediante la Directiva IP/99/572 de la Comisión Europea), en Colombia solamente hasta el año anterior, con la expedición de la Ley 1968 de 11 de julio de 2019, prohibió su uso y creó una política pública para la sustitución de esta sustancia. Al respecto, Observatorio de Redes de Acción Colectiva (2018).

75 Este tipo de argumento pone en consideración el papel de nuestras intuiciones morales en el diseño de nuestra legislación y contextualiza el derecho de la responsabilidad civil a uno en el que las instituciones regulativas son deficientes. PrIEL $(2019, \S 50$ y 51) se pregunta por un tipo de argumento tal para ser desarrollado a raíz de los trabajos que discute con Keating. Esta es solo una pequeña contribución a esa discusión. 


\section{BIBLIOGRAFÍA CITADA}

ACCIARRI, Hugo A. (2013): "El análisis económico del derecho de daños. Una aproximación general", en Bernal, Carlos y FABRA, Jorge Luis (eds.), $L a$ filosofia de la responsabilidad civil. Estudios sobre los fundamentos filosófico-jurídicos de la responsabilidad civil extracontractual (Universidad Externado de Colombia), pp. 421-451.

Aristóteles (2003): Ética a Nicómaco (trad. castellana de José Luis Calvo Martínez, Alianza Editorial).

BAMMER, Gabriele (2008): “The Nature of Uncertainty", en BAMmER, Gabriele y Smithson, Michael (eds.), Uncertainty and Risk. Multidisciplinary Perspectives (Earthscan), pp. 289-304.

Beck, Ulrich (1992): Risk Society. Towards a New Modernity (trad. Mark Ritter, Sage Publications).

Coleman, Jules L. (2010): Riesgos y daños (trad. de Diego M. Papayannis, Marcial Pons).

Darwall, Stephen (2006): The Second-Person Standpoint. Morality, Respect and Accountability (Harvard University Press).

DarwaLl, Stephen (2013a): Morality, Authority, and Law. Essays in Second-Personal Ethics I. (Oxford University Press).

Darwall, Stephen (2013b): Honor, History and Relationships. Essays in Second-Personal Ethics II. (Oxford University Press).

Diez Schwerter, José Luis (2016): La acción de daño contingente del artículo 2333 del Código Civil chileno: sus elementos y ámbito de aplicación. Revista de Derecho de la Pontificia Universidad Católica de Valparaíso XLVI, pp. 133 - 153.

Dorfman, Ariel (2014): “Assumption of Risk, After All”. Theoretical Inquiries in Law, Vol. $15 \mathrm{~N}^{\mathrm{o}} 2$. 
Dyson, Matthew y Steel, Sandy (2018): "Risk and English Tort Law", en Dyson, Matthew (ed.) Regulating Risk through Private Law (Intersentia), pp. 23-53.

EpsteIn, Richard (1973): “A Theory of Strict Liability”, The Journal of Legal Studies, Vol. 2 No 1, pp. 151-204.

FERRER, Jordi (2007): La valoración racional de la prueba (Marcial Pons).

FigueroA, Sebastián (2019): Adscripción y reacción. Responsabilidad jurídica $y$ moral desde una perspectiva interpersonal (Marcial Pons).

FinkelsteIn, Claire (2003): “Is Risk a Harm?”, University of Pennsylvania Law Review, Vol. 151, pp. 963-1001.

FletCHER, George (1972): "Fairness and Utility in Tort Theory", Harvard Law Review, Vol. 85, pp. 537-573.

GaLINDO, Jorge (2015): "El concepto de riesgo en las teorías de Ulrich Beck y Niklas Luhmann”, Acta Sociológica, Vol. 67, pp. 141-164.

GidDENS, Anthony (1999): "Risk and Responsibility", Modern Law Review, Vol. $62 \mathrm{~N}^{\mathrm{o}} 1$, pp. 1-10.

GoldBERG, John y Zipursky, Benjamin (2002): “Unrealized Torts”, Valparaiso Law Review, Vol. 88 No 8, pp. 1.625-1.719.

HaAck, Susan (2014): Evidence Matters. Science, Proof and Truth in the Law (Cambridge University Press).

HaAck, Susan (1982): Filosofía de las lógicas (trad. Amador Antón, Ediciones Cátedra).

Hacking, Ian (1975): The Emergence of Probability: A Philosophical Study of Early Ideas about Probability, Induction and Statistical Inference (Cambridge University Press).

HÁJEK, Alan (2019): "Interpretations of Probability”, en ZALTA, Edward N. (ed.), The Stanford Encyclopedia of Philosophy (Fall 2019 ed.), recuperado de www.plato.stanford.edu/archives/fall2019/entries/probability-interpret/ (última vez consultado el 3 de junio de 2020). 
Hansson, Sven Ove (2018): "Risk", en Zalta, Edward N. (ed.), The Stanford Encyclopedia of Philosophy (Fall 2019 ed.), recuperado de https://plato. stanford.edu/archives/fall2018/entries/risk/ (última vez consultado, 3 de junio de 2020).

Hansson, Sven Ove (2009): "Risk and Safety in Technology", en MeiJers, Anthonie (ed.), Handbook of the Philosophy of Science. Philosophy of Technology and Engineering Sciences, Vol. 9 (Elsevier), pp. 1069-1102.

Hansson, Sven Ove (2007): "Risk and Ethics. Three Approaches", en Lewens (ed.), Risk. Philosophical Perspectives (Routledge), pp. 21-35.

HuRD, Heidi M. (1994): "What in the World Is Wrong”, Journal of Contemporary Legal Issues, Vol. 5, pp. 157-216.

HuRD, Heidi M. (1996): “The Deontology of Negligence”, Boston University Law Review, Vol. 76, pp. 249-272.

JAmes, Aaron (2017): “The Distinctive Significance of Systemic Risk”, Ratio Juris, Vol. $30 \mathrm{~N}^{\mathrm{o}}$ 3, pp. 239-258.

Keating, Gregory (2003): "Irreparable Injury and Extraordinary Precaution: The Safety and Feasibility Norms in American Accident Law", Theoretical Inquiries in Law, Vol. $4 \mathrm{~N}^{\circ}$ 1, pp. 1-88.

Keating, Gregory (2012): "Is the Role of Tort to Repair Wrongful Losses?", en Nolan y Robertson (eds.), Rights and Private Law (Hart Publishing), pp. 491-523.

Keating, Gregory (2012): “The Priority of Respect over Repair". Legal Theory 18, pp. 293-337.

Keating, Gregory (2018): "Principles of Risk Imposition and the Priority of Avoiding Harm", Revus, Vol. 36, recuperado de http://journals.openedition. org/revus/4406 (última vez visitado el 3 de junio de 2020).

KNIGHT, Frank (1971): Risk, Uncertainty and Profit (University of Chicago Press).

Koziol, Helmut (2017): Harmonisation and Fundamental Questions of European Tort Law (Jan Sramek Verlag). 
LANDes, William M. y Posner, Richard A. (1983): "Causation in Tort Law: An Economic Approach”, Journal of Legal Studies, Vol. 12, pp. 109-134.

Luhmann, Niklas (1991): Soziologie des Risikos (De Gruyter Studienbuch).

Luhmann, Niklas (1993): Risk: A Sociological Theory (Walter de Gruyter).

Moore, Michael (2011): Causalidad y Responsabilidad (trad. al castellano de Schleider, Marcial Pons).

Nolan, Donal (2013): "Deconstructing the Duty of Care", Law Quarterly Review, Vol. 129, pp. 559-588.

OBERDIEK, John (2017): Imposing risk. A normative Framework (Oxford University Press).

OBSERVATORIO de Redes y ACCIÓn COLECTIVA ORAC (2018): Asbesto: ¿un peligro silencioso? Parte 1: La prohibición y la regulación del uso del asbesto en el mundo (Universidad del Rosario).

OLIVÉ, León (1981): “El concepto de probabilidad”, Crítica: Revista Hispanoamericana de Filosofía, Vol. 13 No 37, pp. 29-56.

Papayannis, Diego (2014): Comprensión y justificación de la responsabilidad extracontractual (Marcial Pons).

PaPAYANNIS, Diego (2016): El derecho privado como cuestión pública (Universidad Externado de Colombia).

Perry, Stephen (1995): "Risk, Harm and Responsibility", en Owen, David (ed.), Philosophical Foundations of Tort Law (Oxford University Press), pp. 321-346.

Perry, Stephen (2003): "Harm, History, and Counterfactuals", San Diego Legal Review, Vol. 40, pp. 1283-1313.

PlacANI, Adriana (2016): "When the Risk of Harm Harms", Law and Philosophy, Vol. $36 \mathrm{~N}^{\mathrm{o}} 1$, pp. $77-100$.

Porat, Ariel y Stein, Alex (2001): Tort Liability under Uncertainty (Oxford University Press). 
Posner, Richard A. (1972): "A Theory of Negligence”. The Journal of Legal Studies, 1(1), pp. 29-96.

Priel, Dan (2019): “Do Societies Prioritize Harm Prevention?”, Revus [Online], Vol. 37, recuperado de http://journals.openedition.org/revus/5026 (última vez consultado el 3 de junio de 2020).

Raz, Joseph (1986): The Morality of Freedom (Oxford University Press).

RAZ, Joseph (1996): Ethics in the Public Domain (Clarendon).

RENN, Ortwin (1992): “Concepts of Risk: A Classification”, en KRIMSKY y GoldING (eds.), Social Theories of Risk (Westport), pp. 53-79.

RIESCH, Hauke (2012): "Levels of Uncertainty”, en RoESER et al. (eds.) Handbook of Risk Theory (Springer), pp. 87-112.

RIPSTEIN, Arthur y ZIPURSKY, Benjamin (2001): "Corrective Justice in an Age of Mass Torts", en Postema, Gerald (ed.), Philosophy and the Law of Torts (Cambridge University Press), pp. 214-249.

Roisman, Anthony Z. y WolfF, Alexander (2010): "Trespass by Pollution: Remedy by Mandatory Injunction”, Fordham Environmental Law Review, Vol. 21 $\mathrm{N}^{\mathrm{o}}$ 1, pp. 157-192.

SCHROEDER, Christopher (1990): "Corrective Justice, Liability for Increasing Risks”. UCLA Law Review, Vol. 38, pp. 143-162.

SheInMAN, Hanoch (2003): "Tort Law and Corrective Justice", Law and Philosophy, Vol. $22 \mathrm{~N}^{\mathrm{o}} 1$, pp. 21-73.

SHIFFrin, Seana (2012): "Harm and its Moral Significance", Legal Theory, Vol. $18 \mathrm{~N}^{\mathrm{o}} 3$, pp. 357-398.

Strawson, Peter (1995): Libertad y Resentimiento (trad. Acero, J., Paidós).

TAYlor, Peter (2012): “The Mismeasure of Risk", en RoESER et al. (eds.), Handbook of Risk Theory (Springer) pp. 442-476.

TichÝ, Lubos (2013): "On Prevention in Law: Special Focus on Tort Law", en TichÝ y Hrádek (eds.), Prevention in Law (Prague Centrum Právni Komparatistiky Právnické Fakulty Univerzity Karlovy v Praze), pp. 9-29. 
Toulmin, Stephen (1958): The Uses of Argument (Cambridge University Press).

TurTon, Gemma (2015): "Risk and the Damage Requirement in Negligence Liability”, Legal Studies, Vol. 35 No 1, pp. 75-95.

VAN DE Poen, Ibo y Nihlén, Jessica (2018): "Risk and Responsability", en ROESER et al. (eds.), Handbook of Risk Theory (Springer), pp. 877-908.

VARGAS-TINOCO, Alexander (2018): "El valor de no dañar: de la responsabilidad civil a la prevención", en PaPayannis y Pereira (eds.), Filosofía del Derecho Privado (Marcial Pons), pp. 327-357.

Wallace, R. Jay (2007): "Reasons, Relations, and Commands: Reflections on Darwall”, Ethics, Vol. 118 No 1, pp. 24-36.

Wallace, R. Jay (2019): The Moral Nexus (Princeton University Press).

WeINRIB, Ernest (1992): "Non-relational Relationships: A Note on Coleman's New Theory", Iowa Law Review, Vol. 77, pp. 445-448.

WeINRIB, Ernest (2017): La idea de derecho privado (trad. Castellana de Eze Páez, Marcial Pons)

WeINRIB, Ernest (2016): “Causal Uncertainty”, Oxford Journal of Legal Studies, Vol. $36 \mathrm{~N}^{\mathrm{o}} 1$, pp. 135-164.

WolfF, Jonathan (2006): "Risk, Fear, Blame, Shame and The Regulation of Public Safety", Economics and Philosophy, Vol. 22, pp. 409-427.

Wood, Oliver (1964): "Evolution of the Concept of Risk", The Journal of Risk and Insurance, Vol. $31 \mathrm{~N}^{\mathrm{o}} 1$, pp. 83-91.

Wright, Richard (1985): “Causation in Tort Law", California Law Review, Vol. $73 \mathrm{~N}^{\mathrm{o}} 6$, pp. 1735-1828.

Wright, Richard (1988): “Causation, Responsibility, Risk, Probability, Naked Statistics, and Proof: Pruning the Bramble Bush by Clarifying the Concepts", Iowa Law Review, Vol. 73, pp. 1001-1077.

Wright, Richard (2008): "Liability for Possible Wrongs: Causation, Statistical Probability and the Burden of Proof", Loyola of Los Angeles Law Review, Vol. 41, pp. 1295-1344. 
ZoRZETTO, Silvia (2018): Concepto de riesgo y principio de precaución (Ediciones Olejnik). 


\section{JURISPRUDENCIA CITADA}

\section{Estados Unidos:}

Restatement (Second) of Torts $§ 158$ (1965)

Ayers v. Jackson Township, 525 A.2d 287, (N.J. 1987)

\section{Reino Unido:}

Redland Bricks v. Morris [1970] AC 652, 664.

McGhee v. National Coal Board, [1972] 3 All E.R. 1008, 1 W.L.R. 1

Lloyd v. Symonds \& Ors Respondent [1998] EWCA Civ 511

Fairchild v Glenhaven Funeral Services Ltd. [2002] UKHL 22

Birmingham Development Company Ltd v. Tyler [2008] EWCA Civ 859

London Borough of Islington v. Margaret Elliott, Peter Morris [2012] EWCA Civ 56

Mbasogo v. Logo Ltd [2006] EWCA Civ 1370 